\title{
Corrigendum: Reactive oxygen species act through p38 MAPK to limit the lifespan of hematopoietic stem cells
}

Keisuke Ito, Atsushi Hirao, Fumio Arai, Keiyo Takubo, Sahoko Matsuoka, Kana Miyamoto, Masako Ohmura, Kazuhito Naka, Kentaro Hosokawa, Yasuo Ikeda \& Toshio Suda

Nat. Med. 12, 446-451 (2006); published online 26 March 2006; corrected after print 7 January 2010

In the version of this article initially published, two micrographs in Figure $2 c$, corresponding to the conditions $\mathrm{BSO}(-)$ Lineage ${ }^{-}$and $\mathrm{BSO}(+)$ Lineage $^{-}$, were incorrect. These micrographs have been replaced with the correct micrographs in the HTML and PDF versions of the article.

\section{Corrigendum: Modulating hedgehog signaling can attenuate the severity of osteoarthritis}

Alvin C Lin, Brian L Seeto, Justyna M Bartoszko, Michael A Khoury, Heather Whetstone, Louisa Ho, Claire Hsu, Amanda S Ali \& Benjamin A Alman

Nat. Med. 15, 1421-1425 (2009); published online 15 November 2009; corrected after print 7 January 2010

In the version of this article initially published, the author name Amanda S. Ali was incorrect. The correct name is S. Amanda Ali. The error has been corrected in the HTML and PDF versions of the article.

\section{Erratum: Drugs in the headlines}

Victoria Aranda

Nat. Med. 15, 1353 (2009); published online 4 December 2009; corrected after print 8 December 2009

In the version of this article initially published, the sentence "However, it failed in trials against early-stage colon cancers, and reports suggested that it might promote cancer metastasis in certain circumstances" was incorrect. Although it has been suggested that other strategies targeting blood vessel growth factors might promote tumor growth, this has not been specifically shown for Avastin. The text should read "However, it failed in trials against early-stage colon cancers. Reports have also suggested that targeting blood vessel growth factors might promote cancer metastasis in certain circumstances." The error has been corrected in the HTML and PDF versions of the article.

\section{Erratum: Drugs in the headlines}

Victoria Aranda

Nat. Med. 15, 1353 (2009); published online 4 December 2009; corrected after print 8 December 2009; corrected after print 11 December 2009

In the version of this article initially published, it was stated that Denosumab had gained approval for use in osteoporosis treatment, when in fact it had only been recommended for approval. Additionally, the approval of its preventive use was recommended against until further data are available. The text should read "Several clinical trials tested this antibody, which counteracts bone loss. Although a US Food and Drug Administration committee recommended its approval for osteoporosis treatment, it recommended against approval of its preventive use-and its use to treat bone loss in certain cancer cases - until more data are available." The error has been corrected in the HTML and PDF versions of the article. 